\title{
Microphallidae, Trematoda : XLVIII. - Quatre MARITREMA DU Groupe EROliae parasites d'OiseauX aUstraliens
}

\author{
DEBLOCK S.* \& CANARIS A.G.**
}

\begin{abstract}
Summary : MicrophallidAe, TREMATOdA : XLVIII. - Four Maritrema of the EROliae group, parasites of Australian birds The authors describe and illustrate four trematodes from Australia belonging to the genus Maritrema Nicoll. A) From Tasmania, $M$. spinosulum n.sp., of Charadrius ruficapillus is a small species (250-350 $\mathrm{mm}$ ) with vitelline glands in the shape of a horseshoe and its equatorial ribbon with ramifications, and an evaginated curved cirrus $47 \mu \mathrm{m}$ long and spined (thin spines of 1-4,5 $\mu \mathrm{m}$ long projecting from hemispheric basel. A species from Arenaria interpres $400 \mu \mathrm{m}$ long bears cirrus spines like those above but the spines are longer; the species seems more similar to the type of M. eroliae Yamaguti, 1939 than to M. eroliae sensu Bridgman et al., 1972; this latter species could be M. urarayasensis Ogata, 1951. B) From Queensland, M. rubeum n.sp. of Charadrius mongolus and Calidris acuminata is 400 um long, with ring of vitelline glands closed posteriorly, a cirrus $100 \mu \mathrm{m}$ long covered with strong trianguliform spines of $6,5-11 \mu \mathrm{m}$; this species has no counterpart in literature. Maritrema sp. from C. acuminata, $200 \mu \mathrm{m}$ long with a spinose cirrus (spines of about $2 \mu \mathrm{m}$ long) is like M. macropharynga Ke, 1976 but with a smaller pharynx. Maritreminoides patulum Cable et al., 1960 becomes a new species, Maritrema caribbeanse.
\end{abstract}

KEY WORDS : Trematodes, Microphallidae, Maritrema, birds, Australia, taxonomy, Maritrema spinosulum, Maritrema rubeum, Maritrema eroliae, Maritrema caribbeanse.

\section{INTRODUCTION}

$\mathrm{L}$ es auteurs décrivent et illustrent quatre espèces de trématodes Microphallidae du genre Maritrema Nicoll, 1907 faisant partie du groupe Eroliae défini par Deblock et Canaris, 1992 (cirres évaginés volumineux, courbés et épineux). Deux espèces proviennent de Tasmanie; deux autres du Queensland (Australie). L'identification de $M$. eroliae Yamaguti est précisée et l'identité de Maritreminoides patulum Cable et al., 1960 (nec Coil, 1955) est reconsidérée.

\section{MATÉRIEL ET MÉTHODE}

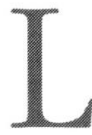

e premier lot de trématodes est récolté par A.G. Canaris en 1993 en Tasmanie; le second lot vient du Queensland (Australie) décrit initiale-

* Parasitologie, Faculté de Pharmacie, BP 83, F-59006 Lille Cedex. ** University of Texas at El Paso, PO Box 1479, Hamilton, USA, MT 59840-1479.

\section{Résumé :}

Les auteurs décrivent et illustrent quatre trématodes d'Australie du genre Maritrema Nicoll. A) En Tasmanie, M. spinosulum n.sp. de Charadrius ruficapillus présente une petite taille $(250-350 \mu \mathrm{m})$, des vitellogènes en fer à cheval à bande équatoriale ramifiée, un cirre évaginé courbé long de $47 \mu m$ et épineux (fines épines de I à 4,5 $\mu \mathrm{m}$ implantées sur une base hémisphérique). Une espèce d'Arenaria interpres, longue de $400 \mu \mathrm{m}$ et aux épines du cirre analogues mais plus grandes, parait mieux conforme à la redescription du type de M. eroliae Yamaguti, 1939 qu'à l'espèce M. eroliae sensu Bridgman et al., 1972, plutôt identifiable à M. urayasensis Ogata, 1951. B) Au Queensland M. rubeum n. sp., de Charadrius mongolus et de Calidris acuminata est longue de $400 \mu \mathrm{m}$ avec des vitellogènes en anneau fermé et un cirre long de $100 \mu \mathrm{m}$ armé de fortes épines trianguliformes $(6,5$ à $11 \mathrm{\mu m})$; l'espèce n'a pas d'équivalent connu. Maritrema sp. de C. acuminata, longue de $200 \mu \mathrm{m}$, au cirre armé d'épines de $2 \mu \mathrm{m}$, s'apparente à $M$. macropharynga $\mathrm{Ke}, 1976$ avec un pharynx moins volumineux. Maritreminoides patulum Cable et al., 1960 est individualisé sous le nom de Maritrema caribbeanse $n$. sp.

MOTS CLÉS : Trematoda, Microphallidae, Maritrema, Oiseaux, Australie, taxonomie, Maritrema spinosulum, Martrema rubeum, Maritrema eroliae, Maritrema caribbeanse.

ment par Deblock \& Pearson, 1968 sous un nom collectif ( $M$. eroliae Yamaguti) recouvrant en fait trois taxons différents après un nouvel examen critique. La méthode d'étude résulte des opérations courantes en helminthologie descriptive (récolte puis observation en microscopie à lumière transmise de parasites fixés, colorés et montés au baume; dessins tracés à la chambre claire). Les mesures en micromètres expriment hauteurs $\times$ largeurs.

\section{MARITREMA SPINOSULUM n. sp.}

Hôte définitif : Charadrius ruficapillus, redcapped dotterel (deux hôtes). Tube digestif (intestin grêle ou cæca selon l'hôte). Lieu géographique : King Island, Tasmania (Australie). Huit exemplaires adultes récoltés le 2 mars 1993 (A.G. Canaris).

\section{Description (fig. 1)}

Corps de contour ovale allongé et de petite taille : 300328-350 × 136-170-197 $\mu \mathrm{m}$. Rapport longueur/largeur $=0,5$. Tégument fin. Ventouse orale (VO) : $29 \times 32 \mu \mathrm{m}$. 
Pré-pharynx : $10 \mu \mathrm{m}$. Pharynx : $24 \times 24 \mu \mathrm{m}$. Ventouse ventrale $(\mathrm{VV}): 34 \times 34 \mu \mathrm{m}$. $\mathrm{VO} / \mathrm{VV}=1 / 1,2$ ou 0,8 . La VV se situe légèrement au-delà du milieu du corps. CEsophage : $40 \mu \mathrm{m}$. Cæeca égaux: $95 \times 15-16 \mu \mathrm{m}$; leurs fonds acétabulaires sont adjacents aux bords corporels et non en contact avec les vitellogènes transverses. Testicules postéro-latéraux symétriques, ovalaires, égaux $(52 \times 38 \mu \mathrm{m})$, situés en arrière des anses vitellogènes. Poche du cirre fusiforme longue à paroi musculaire fine, située entre cæcum et vitellogène à partir du bord aporal du corps, contournant le bord antérieur de la VV avant d'aborder le pore génital para-acétabulaire gauche. Taille : 130-146-186 × $32 \mu \mathrm{m}$. Longueurs poche du cirre/corps $(\mathrm{PdC} / \mathrm{LC})=0,44$. Vésicule séminale fusiforme allongée égale à la mi-longueur de la poche du cirre. Cirre invaginé non observé. Cirre évaginé courbé et massif, long de 30-47-55 × $19 \mu \mathrm{m}$ de diamètre, revêtu d'épines fines régulièrement implantées sur toute sa surface et de morphologies semblables; les épines distales sont plus courtes (1 à $2 \mu \mathrm{m}$ ) que les épines proximales $(4,5 \mu \mathrm{m})$, et leurs tailles sont progressivement croissantes (fig. 2). Ovaire médian, sous-acétabulaire, trilobé, de $62 \times 37 \mu \mathrm{m}$. Vitellogènes linéaires typiques du genre, disposés en fer à cheval; l'écartement des extrêmités postérieures libres des guirlandes est égal à 60-115 $\mu \mathrm{m}$ (moyenne $100 \mu \mathrm{m}$ ). Les vitellogènes dorsaux équatoriaux constituent de façon aléatoire de courtes ramifications antérieures ou postérieures (de 20 à $30 \mu \mathrm{m}$ d'extension), au nombre de un ou deux par côté (fig. 3). Insertion terminale ou subterminale des courts vitelloductes. Anses utérines postacétabulaires ne dépassant pas antérieurement la limite du fond des cæca. Métraterme de $40 \times 15 \mu \mathrm{m}$ à paroi interne villeuse. CEufs abondants de 11,5- 13 -16 $\mu \mathrm{m}$ $\times 7-9 \mu \mathrm{m}$. Atrium génital discret et simple. Vessie en Y aux branches paires longues $(100 \mu \mathrm{m})$ côtoyant le bord interne des testicules; branche impaire courte. Solénocytes non détectables.

\section{Discussion}

Le Maritrema fait partie des espèces du groupe Eroliae au corps long de moins de $400 \mu \mathrm{m}$, et au nombre de cinq : quatre dans la zone du Pacifique asiatique et une en mer des Caraibes. M. borneoense Fischthal et Kuntz, 1973 s'en distingue par des oufs de grande taille (26-29 $\mu \mathrm{m})$ et une armature du cirre d'épines longues (6-7 $\mu \mathrm{m} \times 3$ à $4 \mu \mathrm{m}$ à la base) dont l'implantation n'est pas précisable sur les types observés. M. macropharynga Ke, 1976 s'en distingue par un pharynx d'un diamètre de $44 \times 40 \mu \mathrm{m}$ approchant celui de l'acétabulum, une poche du cirre transverse plus courte $(123 \mu \mathrm{m})$ que le cæecum aporal $(140 \mu \mathrm{m})$ et n'atteignant pas le bord du corps; la spinulation du cirre n'est pas décrite. M. minuta Ke, 1976 est l'espèce dont les mensurations sont les plus voisines de celles de $M$. spinosulum avec un cirre de $65 \times 22 \mu \mathrm{m}$ dont la spinulation n'est pas décrite. La comparaison avec M. jilinensis Zhong et al., 1988 est aléatoire, car cette espèce parait composite : la métacercaire figurée (400 $\mu \mathrm{m})$ est plus grande que l'adulte $(266 \mu \mathrm{m})$, et sa morphologie différente (rapport ventousaire, poche du cirre plus longue que le caecum homologue, vitellogène en anneau fermé); l'illustration présente des anomalies (œufs figurés trop petits et pourvus d'un miracidium) ou des omissions (spinulation symbolique du cirre). L'espèce $M$. patulus américaine pose aussi un problème d'interprétation après observation des divers spécimens de collection de l'US NM. Le cirre de Maritreminoides patulum Cable et al., 1960 nec Coil, 1955 est pourvu d'une armature dense d'épines basales, longues de 4 à $5 \mu \mathrm{m}$ et rassemblées en un bouquet distal, alors que le long canal éjaculateur d'amont paraît verruqueux et glabre. Le cirre cylindrique évaginé de Maritrema patulus Coil, 1955 est long de $70 \mu \mathrm{m}$ et glabre, contrairement à l'affirmation du texte. Les deux espèces ne sont donc pas synonymes; il est proposé que $M$. patulum C., C. et B. prenne le nom de Maritrema caribbeanse n. sp. Par ailleurs, aucune des espèces mentionnées en discussion ne présente des vitellogènes équatoriaux ramifiés.

L'espèce de Tasmanie sera individualisé sous le nom de Maritrema spinosulum n. sp. (à épines clairsemées) dans l'attente d'une description plus appropriées des espèces citées.

Remarque. - Quatre spécimens de Maritrema parasites de Charadrius mongolus, déterminés à tort comme $M$. eroliae Yamaguti in Deblock et Pearson, 1968, sont désormais rattachés à l'espèce spinosulum; le corps est long de 260 à $330 \mu \mathrm{m}$, et le cirre invaginé (CI) de $60 \mu \mathrm{m}$. Le rapport $\mathrm{CI} / \mathrm{PdC}=0,38$. Le cirre est tapissée d'un revêtement continu de fines épines serrées longues de 1 à $4,5 \mu \mathrm{m}$, avec l'apparence d'un fin velours en profil et d'une fine ponctuation en plan (fig. 7A). CEufs longs de 11 à $18 \mu \mathrm{m}$.

\section{MARITREMA EROLIAE Yamaguti, 1939}

Hôte définitif : Arenaria interpres (deux hôtes), Charadrius ruficapillus (deux hôtes). Tube digestif (grêle ou cæca selon l'hôte). King Island, Tasmania; $2 \times 2$ exemplaires adultes (438 et $450 \mu \mathrm{m}$, et 380 et $400 \mu \mathrm{m}$ de long), récoltés le 7 mars 1993 (A.G. Canaris)

\section{DesCription (fig. 4).}

Corps de taille moyenne : $410 \times 220$. VO : $38 \times 37$. VV : $38 \times 37$. Pharynx : $25 \times 25$. Poche du cirre : 175 $\times$ 37. PC/LC : 0,42. Cirre évaginé courbé en croissant troncônique long de $80 \times 32$ de diamètre à sa base; à l'exception d'une zone glabre de la petite courbure, le cirre est armé d'épines de tailles progressivement 

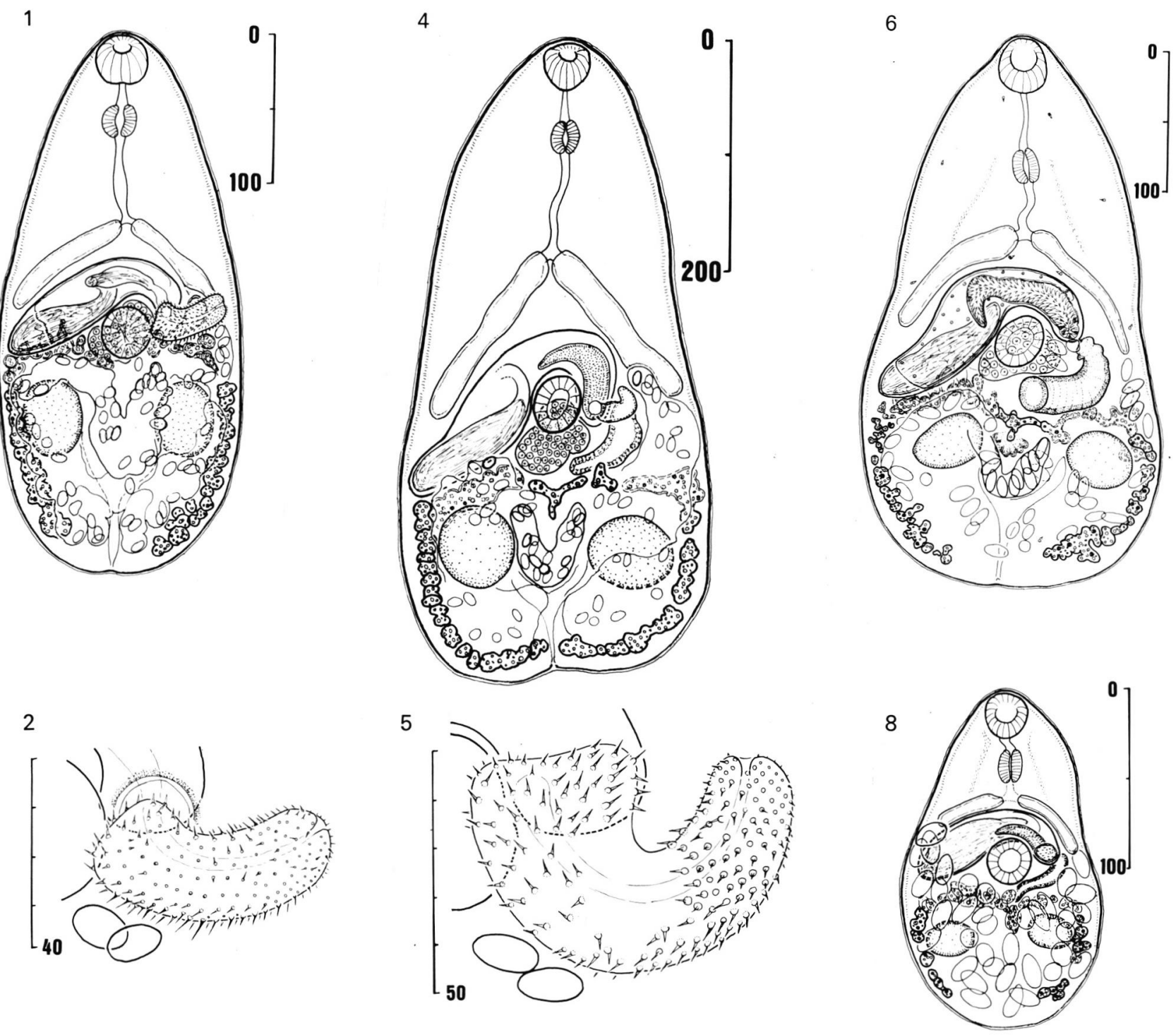

A

3

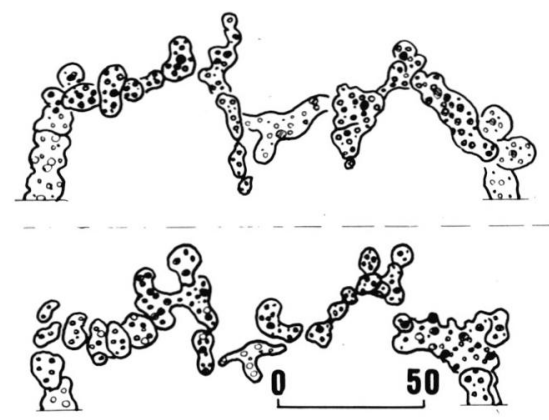

7

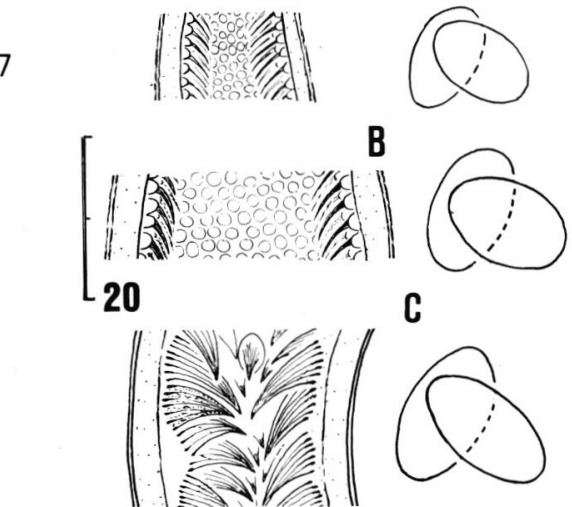

Fig. 1. - Maritrema spinosulum n. sp. Charadrius ruficapillus, Tasmanie. Adulte, vue ventrale.

Fig. 2. - M. spinosulum. Cirre évaginé.

Fig. 3. - M. spinosulum. Deux exemples de ramifications des vitellogènes transverses.

Fig. 4. - Maritrema eroliae Yamaguti, 1939. Arenaria interpres, Tasmanie. Adulte, vue ventrale.

Fig. 5. - M. eroliae, Tasmanie. Cirre évaginé.

Fig. 6.- Maritrema rubeum n. sp. Calidris acuminata, Queensland (Australie). Adulte, vue ventrale.

Fig. 7. - Apparence semi-schématique de la spinulation des cirres invaginés de trois espèces de Maritrema du groupe Eroliae, parasites de Charadrius mongolus (Queensland) et deux oufs mûrs figurés à droite : A) M. spinosulum n. sp. B) M. eroliae Yamag., 1939. C) M. rubeum n. sp.

Fig. 8. - Maritrema sp. de Cal. acuminata (Queensland) : adulte, vue ventrale 
croissantes de 1,5 à $5 \mu \mathrm{m}$, fines et acérées, reposant sur une base circulaire et hémisphérique de 1-2,5 $\mu \mathrm{m}$ de diamètre (fig. 5). Le rapport $\mathrm{CI} / \mathrm{PdC}=0,45$; la lumière du canal du cirre invaginé est tapissée d'un revêtement continu de fines épines dont les contours juxtaposés des bases arrondies évoquent l'apparence d'un " nid d'abeille " dès lors qu'ils sont perçus de face (fig. 7B). Anneau vitellin fin et complet. CEufs longs de $16-19 \mu \mathrm{m}$.

\section{Discussion}

Anatomie et dimensions de l'espèce correspondent à celles des types de M. eroliae Yamaguti, 1939 [in Deblock, 1975 : corps de $490 \times 237$ (extrêmes : 400550). VO : $37 \times 39$. VV : $43 \times 40$. Pharynx : $29 \times 21$. Poche du cirre : $242 \times 58$. PC/LC : 0,49. Cirre invaginé pourvu d'épines intra-luminales morphologiquement homogènes et de petites tailles. Anneau vitellin fermé. CEufs : $17-19 \mu \mathrm{m}]$. Il apparaît présentement aux auteurs que l'identité de l'espèce décrite sous le nom d'eroliae par Bridgman et al., 1972 corresponde davantage à M. urayasensis Ogata, 1951 qu'à eroliae, en fonction de la seconde hypothèse formulée par Deblock, 1975; la spinulation homogène du cirre d'eroliae s'oppose à la spinulation hétérogène du cirre d'urayasensis [grosses épines triangulaires basales - donc pseudoatriales sur le cirre invaginé - disséminées au sein de petites épines côniques, seules présentes distalement (selon Deblock, 1975 d'après les spécimens récoltés par Bridgman et al., 1972)].

L'hétérogénéité du matériel identifié comme "Maritrema eroliae Yamaguti, 1939 " par Deblock et Pearson, 1968 incite à réviser la position taxonomique des divers spécimens de ce lot de Microphallidés, parasites du tube digestif de Charadrius mongolus et de Calidris acuminata en provenance de Raby Bay, Queensland, et récoltés le 31 janvier 1957 (J.C. Pearson).

\section{MARITREMA RUBEUM n. sp.}

Matériel de description : une quarantaine d'exemplaires (dont quelques métacercaires juste dékystées à œufs utérins rares). Mesures de cinq individus de taille moyenne issus des deux hôtes sus-nommés.

\section{DESCRIPTION (fig. 6)}

Corps linguiforme de $370 \times 224 \mu \mathrm{m}$ (extrêmes : 346 et 387; mais 285 à $435 \mu \mathrm{m}$ dans le lot des 40 exemplaires observés). Ventouse orale : 35 . Ventouse ventrale $: 32 . \mathrm{VO} / \mathrm{VV}=1,19$. Pré-pharynx $: 28$. Pharynx : $24 \times 19$. CEsophage : 24. Cæca à fonds acétabulaires : 120 et 100 . Poche du cirre (PdC) fusiforme allongée $(175 \times 42 \mu \mathrm{m})$ et courbée en crochet antérieur contournant l'acétabulum jusqu'au pore génital para-acétabulaire senestre. Rapport longueurs de la poche du cirre à celle du corps $(\mathrm{PdC} / \mathrm{LC})=0,47$. Vésicule séminale : $80 \times 25$. Pars prostatica et canaux afférents non repérables. Canal du cirre invaginé (CI) long de $106 \mu \mathrm{m}$. Rapport $\mathrm{CI} / \mathrm{PdC}=0,6$. Ce canal est armé de fortes et nombreuses épines triangulaires en épines de rosier, toutes semblables à la taille près $(6,5$ à $11 \mu \mathrm{m})$, implantées sur une base ovalaire et ponctuée dont les plus grandes mesurent $6,5 \times 5,5 \mu \mathrm{m}$ de diamètre; les ponctuations correspondent à l'épaississement basal de 9 à 15 fins filaments parcourant la longueur de l'épine pour converger vers sa pointe (fig. 7C). Cirre non observé évaginé. Ovaire médian non mesurable. Métraterme développé : $76 \times 22$. Testicules postéro-latéraux, plutôt petits, de $32 \times 36$ et $39 \times 41$. Vitellogènes en anneau fermé ou sub-fermé postérieurement (écartement des extrêmités libres des guirlandes égal à 45-80 $\mu \mathrm{m}$ ). Follicules de $8 \times 10 \mu \mathrm{m}$ de diamètre environ. Vessie en $\mathrm{Y}$ à branches paires longues $(90 \mu \mathrm{m})$ et intertesticulaires. CEufs abondants dans les distomes âgés, longs de 16 à $22 \mu \mathrm{m}$ (moyenne 18-19).

\section{Discussion}

Aucune espèce de Maritrema du groupe Eroliae au corps inférieur à $400 \mu \mathrm{m}$ de long ne présente la structure anatomique du cirre invaginé décrite chez Maritrema rubeum n. sp. ("rubeum " : en rapport avec les ronces). La forme des épines ne se confond pas avec celle des espèces à épines fines à base hémisphérique (comme chez M. eroliae Yamaguti; voir ci-dessus). Le corps de M. magnicirrus Belop., 1952 mesure près de $500 \mu \mathrm{m}$ et le canal du cirre invaginé présente là l'image de Maritrema caribbeanse (Cable et al., 1960) nov. comb., ou de M. kitanensis Shibue, 1953, voire encore de M. papillorobusta Ke, 1976], des épines de tailles inhomogènes $(7-10 \mu \mathrm{m}$ au niveau du bouquet des épines distales et $3,5 \mu \mathrm{m}$ au niveau des proximales).

\section{MARITREMA sp.}

Deux exemplaires d'un très petit Maritrema du groupe Eroliae parasitent un Calidris acuminata du Queensland (fig. 8). Corps : $192 \times 122$. VO : 24. Pharynx : 22 $\times 14$. CEsophage plus court que le pharynx. Cæca : 37 et 42 . VV : 22. Poche du cirre : $93 \times 27$. Vésicule séminale : $66 \times 26$. Canal du cirre invaginé : 38 , à la paroi couverte d'épines longues de 1 à $2 \mu \mathrm{m}$. Testicules 30 $\times 25$ et $22 \times 27$. Métraterme : 40. CEufs : $17,5-21 \times 10$ 11. $\mathrm{PdC} / \mathrm{LC}=0,48 . \mathrm{CI} / \mathrm{PdC}=0,43$.

\section{DisCussion}

Le petit nombre des spécimens observés empèche d'apprécier les variations de taille de l'espèce qu'ils représentent; le Maritrema se situe au niveau des limites inférieures de M. minuta Ke, 1966 mais il pourrait éventuellement s'identifier aussi à $M$. macropharynga Ke, 1976. 
Dépôt des types, paratypes et spécimens des espèces décrites :

Maritrema spinosulum n. sp. : 1) US National Parasite Collection, Beltsville Maryland : $\mathrm{n}^{\circ} 86785$ (type et paratypes). 2) Museum national d'Histoire Naturelle, Paris : $\mathrm{n}^{\circ} 512$ HF-Tk 30 (paratypes).

Maritrema eroliae Yamag., 1939, USNPC : n 70993.01; 86787.01 et 02 (spécimens de référence d'Australie). Maritrema rubeum n. sp., USNPC : no 70993.03 (syntypes).

\section{RÉFÉRENCES}

DeBlock S. Contribution à l'étude des Microphallidés Travassos, 1920 (Trematoda). XXX. - A propos d'espèces décrites au Japon par S. Yamaguti. C. - Maritrema eroliae et le genre Pseudospelotrema. Annales de Parasitologie Humaine et comparée, 1975, 50, 45-54.

Deblock S. \& Canaris A. Contribution à l'étude des Microphallidae Travassos, 1920 (Trematoda). XLIII. - De six espèces d'Afrique du Sud dont une d'un genre nouveau. Ibid., 1992, 67, p. 204-218.

Deblock S. \& Pearson J.C. Contribution à l'étude des Microphallidae Travassos, 1920 (Trematoda). XV. - De quelques espèces d'Australie dont Pseudospelotrema* anenteron n.sp. Ibid., 1968, 43, p. 457-465. (* erreur typographique pour Pseudolevinseniella).

Reçu le 29 février 1996 Accepté le 13 juillet 1996 